
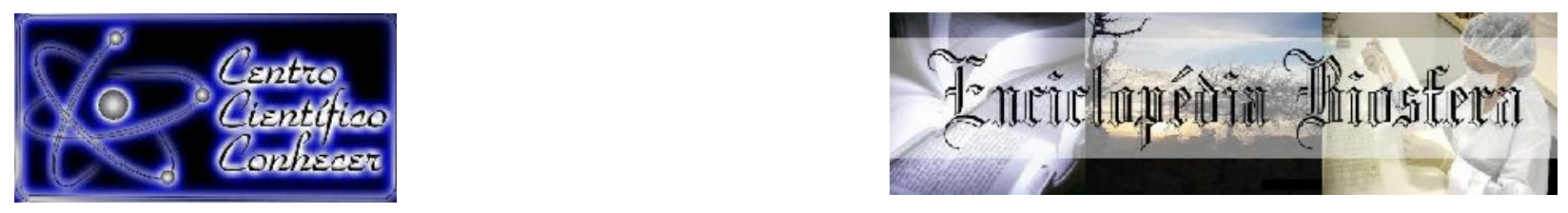

\title{
PRESENÇA DE AMASTIGOTAS DE Leishmania sp. EM SANGUE PERIFÉRICO DE CÃO: RELATO DE CASO
}

Marla Schneider ${ }^{1}$, Marianne Sperduti Moço de Freitas ${ }^{2}$, Jucemara Madel de Medeiros $^{2}$,Fernanda Bernardo Cripa ${ }^{3}$, Luciana Pereira Machado ${ }^{4}$.

1 Pós-graduanda, Universidade Federal Fronteira Sul (UFFS), (marla.schneider.uffs@gmail.com), Realeza-PR, Brasil

2 Acadêmica de Medicina Veterinária, Universidade Federal Fronteira Sul (UFFS), Realeza-PR, Brasil

3 Mestre em Bioenergia, Universidade Federal Fronteira Sul (UFFS), Realeza-PR, Brasil

4 Professora Adjunta, Universidade Federal Fronteira Sul (UFFS), Realeza-PR, Brasil

Recebido em: 06/04/2019 - Aprovado em: 10/06/2019 - Publicado em: 30/06/2019 DOI: 10.18677/EnciBio_2019A103

\begin{abstract}
RESUMO
A leishmaniose visceral canina é uma importante zoonose causadora de sinais clínicos cutâneos e viscerais, que tem o cão como principal reservatório urbano. No Brasil, vários estados são endêmicos para a doença, principalmente no nordeste e norte do país. Com o aumento do deslocamento populacional e de animais de companhia os relatos da doença vêm sendo descritos crescentemente, como é o caso do Paraná que já é considerado endêmico em alguns municípios desde 2013. Descreve-se um caso alóctone de leishmaniose visceral canina diagnosticado em capa leucocitária, com ênfase nas alterações laboratoriais. Um cão da raça ChowChow, macho, de 7 anos proveniente de área endêmica (Tocantins) foi atendido em um Hospital-escola no Paraná. O animal apresentava epistaxe, secreção purulenta periocular; mucosas hipocoradas; claudicação, fraqueza muscular, ataxia e cansaço progressivo, abdômen rígido e álgico a palpação. O hemograma revelou intensa pancitopenia $\left(1,68 \times 10^{6}\right.$ eritrócitos $/ \mu \mathrm{L} ; 1.800$ leucócitos totais $/ \mu \mathrm{L}$ e 29.000 plaquetas $/ \mu \mathrm{L})$ e discreta hipoproteinemia $(5,8 \mathrm{~g} / \mathrm{dL})$ e estruturas compatíveis com Anaplasma sp em plaquetas. Os valores de alanina aminotransferase, fosfatase alcalina, uréia e creatinina estavam dentro do intervalo de referência para a espécie. Foi analisada uma lâmina de capa de leucócitos, na qual foram observadas formas amastigotas de Leishmania sp. em neutrófilos e monócitos. Foram notificados os órgãos responsáveis e o tutor optou pela eutanásia.
\end{abstract}

PALAVRAS-CHAVE: capa leucocitária, leishmaniose visceral canina, pancitopenia 


\title{
PRESENCE OF LEISHMANIA sp AMASTIGOTES IN PERIPHERAL BLOOD OF DOGS: CASE REPORT
}

\begin{abstract}
Canine Visceral Leishmaniasis (CVL) is an important zoonotic disease responsible for tegumentary and visceral clinical signs where domestic dogs are the main urban reservoir. In Brazil, a huge number of federative states are considered endemic areas for this infection, main lythe North eastern and northern parts of the country. With the rising rate of populacional and pet displacement the reports of the disease occurrence are being described at a crescent basis, that is the case of the federative state of Paraná in which some cities are considered na endemic área since 2013. This article presents a foreign case of canine visceral leishmaniasis diagnosed by buffy coat visualization, emphasizing laboratorial alterations. The dog a male, seven years old, Chow-Chow breed exemplar, was provenient of na endemic area (Tocantins) and attended to the clinical examination in a school hospital in Paraná. The dog presented clinical signs as epistaxis, periocular purulent secretion, pallorof mucous membranes, claudication, muscular weakness, ataxy and progressive tiredness, rigid and álgic abdômen at palpation exam. The hemogram revealed intense pancytopenia $\left(1,68 \times 10^{6}\right.$ erythrocytes/ $\mu \mathrm{L} ; 1.800$ total leukocyte/ $\mu \mathrm{L}$ and 29.000 platelets $/ \mu \mathrm{L})$ and discreet hypoproteinemia $(5,8 \mathrm{~g} / \mathrm{dL})$. The alanine transaminase, alkaline phosphatase, urea and creatinine values were inside the reference parameters for the specie. A buffy coat blood smear was analyzed and was observed amastigote forms of Leishmania spp. in neutrophils and monocytes and structures similar to Anaplasma $\mathrm{sp}$ in platelets. The responsible control organs were notified and the guardian chose for euthanasia.
\end{abstract}

KEYWORDS: leukocyte layer, visceral canine leishmaniasis, pancytopenia

\section{INTRODUÇÃO}

A leishmaniose é considerada uma zoonose importante e está presente na maior parte do Brasil, algumas regiões são de caráter endêmico, como é o caso do nordeste (ROCHA et al., 2018). Os reservatórios podem ser animais domésticos ou selvagens, e em alguns casos, menos comum no Brasil, considerada uma antropozoonose, ou seja, o humano a fonte de infecção (KAMHAWI, 2006). A leishmaniose é classificada em visceral (LV) e tegumentar (LT), causadas por espécies diferentes do parasito e com diferentes apresentações clínicas (FARIA; ANDRADE, 2012).

A leishmaniose visceral (LV) é responsável por alterações em órgãos como o fígado, baço e a medula óssea, e tem como principais patógenos no Velho Mundo as espécies Leishmania donovani e Leishmania infantum (AKHOUNDI et al., 2016). No Novo Mundo o agente é da espécie L. infantum (sinônimo de L. chagasi), com predomínio da forma zoonótica de transmissão na qual o cão é o principal reservatório e desenvolve a doença leishmaniose visceral canina (LVC) (MENDONÇA et al., 2017; OLIVEIRA et al., 2017).

A LVC pode causar uma grande variedade de manifestações clínicas nos cães, desde lesões cutâneas, até doença sistêmica causando a morte do paciente (KAMHAWI, 2006, MELENDEZ-LAZO et al., 2017). Os sinais clínicos mais comuns da LVC são perda de peso, linfadenomegalia (FREITAS et al. 2017; MELENDEZLAZO, et al., 2017), febre, lesões oculares, renais e dermatológicas. A evolução ocorre geralmente de forma crônica (BLAVIER et al., 2001). 
Segundo Blavier et al. (2001) gamopatia monoclonal, insuficiência renal, problemas hemostáticos, colite crônica, alterações cardiovasculares, respiratórias e músculo esqueléticas podem ser encontradas em casos de LVC. Na presença de alterações oculares em áreas endêmicas a leishmaniose deve ser considerada como diagnóstico diferencial, e as principais alterações encontradas por Freitas et al. (2017) foram uveíte, blefarite, ceratite, conjuntivite e ceratoconjuntivite seca. De acordo com Zuben e Donalísio (2016), a doença pode permanecer assintomática, o que dificulta o diagnóstico, mas os cães positivos apresentam em sua maioria parasitos na derme.

A transmissão da leishmaniose ocorre por flebótomos do gênero Lutzomya e Phlebotomus (MAIA; CAMPINO, 2008; OLIVEIRA et al., 2015), fêmeas, que necessitam de alimentação por sangue para maturação dos ovos. $O$ inseto contaminado transmite o parasita durante o repasto sanguíneo e tem maior atividade pela manhã e ao anoitecer (OLIVEIRA et al., 2017).

Para completar o ciclo de vida, o protozoário necessita do flebotomíneo, quando está no vetor, se encontra na forma de promastigotas extracelulares no trato digestivo, e no hospedeiro vertebrado na forma de amastigota intracelular (KAMHAWI, 2006). O ciclo pode variar de seis a nove dias e começa quando o vetor ingere células contendo o parasita na forma amastigota, e no trato gástrico inferior do inseto, a Leishmania sp passa por várias fases até a forma infectante (HANDMAN; BULLEN, 2002; KAMHAWI, 2006).

A morfologia do parasita é modificada conforme a fase do ciclo, e para que ocorra o desenvolvimento este possui adaptações para sobreviver dentro do inseto, entre elas, a secreção de um neuropeptídeo que impede o peristaltismo do intestino e a fixação do parasito para evitar a expulsão. A produção de quitinases que degradam a válvula estomodeal, bem como a proteção à enzimas digestivas do vetor através da secreção de fosfoglicanos pelo parasita também são descritos (KAMHAWI, 2006).

A transfusão sanguínea também é relatada como forma de transmissão da doença, sendo importante o monitoramento dos cães doadores mesmo que assintomáticos, para que não haja disseminação do parasita (FREITAS et al., 2006). Oliveira et al. (2017) detectaram DNA do parasita em tecidos reprodutivos de uma cadela naturalmente infectada e seu embrião, comprovando que é possível ocorrer a transmissão vertical. Em áreas endêmicas os testes sorológicos devem ser parte da triagem e todos os animais devem ser considerados potencialmente infectados (BLAVIER et al. 2001).

Nos cães assintomáticos a parasitemia não é persistente e o parasita instalase no sistema mononuclear fagocitário na forma de amastigotas (FREITAS et al., 2006). O macrófago é a principal célula parasitada no hospedeiro mamífero, fazendo importante papel na imunidade. Em seguida a Leishmania $s p$, utiliza mecanismos para estabelecer a infecção e se alojar em diferentes órgãos (HANDMAN; BULLEN, 2002).

O parasita está presente principalmente nos órgãos linfóides secundários, medula óssea e pele dos animais infectados, sendo descrita também a presença em sistema genital (OLIVEIRA et al., 2015). A apresentação de sinais clínicos ou não dependerá dos mecanismos de defesa genéticos e imunológicos do paciente (FREITAS et al., 2006).

A infecção canina é um importante fator de risco para a doença humana (ROCHA et al., 2018), com relevância na saúde pública, aliada a fatores de risco 
ambientais, urbanização, desmatamento entre outros (ABRANTES et al. 2018). Para o controle dos casos de leishmaniose, é necessário um bom estudo epidemiológico e planejamento das estratégias de controle (ORTIZ; ANVERSA 2015; ROCHA et al., 2018), como a utilização de testes diagnósticos, vacinas nos cães e repelentes contra o vetor (ZUBEM; DONALíSIO 2016).

Segundo Mendonça et al. (2017) para o diagnóstico da leishmaniose canina existem métodos sorológicos como a reação de imunofluorescência indireta (RIFI), ensaio imunoenzimático (ELISA) e testes imunocromatográficos rápidos. Métodos moleculares, como reação em cadeia da polimerase (PCR), imuno-histoquímica, citologia e histologia são recomendados, mas o diagnóstico parasitológico é o padrão ouro (PALTRINIERI et al., 2016).

A observação do parasito na forma amastigota em esfregaço de órgãos, pele íntegra ou com lesões, punção de linfonodos, medula óssea e em amostra de sangue periférico geram um diagnóstico conclusivo (MAIA; CAMPINO, 2008). Porém, a pesquisa microscópica de células infectadas em esfregaços de sangue periférico não é satisfatória, devido à porcentagem de células parasitadas por amastigotas circulantes como neutrófilos, linfócitos e monócitos ser baixa $(<0,5 \%$ dos casos) (GIUDICE; PASSANTINO, 2011).

As formas amastigotas, tem de 2 a $4 \mu \mathrm{m}$ de diâmetro, formato oval ou redondo, citoplasma azul pálido, núcleo relativamente grande e uma projeção em forma de bastão denominada cinetoplasto. $O$ parasito pode ser visto intracitoplasmático em monócitos, neutrófilos e macrófagos ou livres na lâmina corada (MAIA; CAMPINO, 2008).

Alterações como anemia normocítica normocrômica não regenerativa e alterações no leucograma são comumente encontrados em cães positivos para LVC (CORTESE et al., 2011; PALTRINIERI et al., 2016). As alterações leucocitárias são inespecíficas (Da COSTA-VAL et al., 2007). Porém, seguidamente são descritos casos com linfocitose, linfopenia ou eosinofilia, alterações em outras populações de leucócitos são menos comuns (DE TOMMASI et al., 2013).

Em cães com leishmaniose visceral sem infecções concomitantes encontra-se frequentemente trombocitopenia leve a moderada (PALTRINIERI et al., 2016). Em quadros de trombocitopenia acentuada, a co-infecção com outros patógenos transmitidos por vetores (por exemplo, Ehrlichia canis, Anaplasma phagocytophilum ou Anaplasma platys) ou outras possíveis causas de redução da concentração de plaquetas devem ser pesquisadas (CORTESE et al., 2011).

Além disso, a presença de azotemia e alterações na urinálise como diminuição na densidade urinária e proteinúria podem indicar disfunção renal (CÂMARA et al., 2017). Hiperproteinemia, hiperglobulinemia (CÂMARA et al., 2017), hipoalbuminemia, são comumente encontrados nos cães com LVC (GODOY et al., 2017; MELENDEZ-LAZO et al., 2017).

Em casos de animais positivos para leishmaniose visceral, a notificação aos órgãos de saúde é compulsória, o Ministério da Saúde recomenda a eutanásia dos animais, porém desde 2016 o tratamento é permitido por Lei, segundo a NOTA TÉCNICA No 11/2016/CPV/DFIP/SDA/GM/MAPA. A terapia medicamentosa só é permitida, cumprindo corretamente o protocolo de tratamento, utilizando medicação de uso não humano, Milteforan, sendo necessário o acompanhamento clínico veterinário desse paciente por toda a vida. 
O trabalho em conjunto entre Médicos Veterinários e profissionais de saúde humana, é uma importante chave para controle da doença, tanto humana quanto animal, debatido como "Saúde Única" por Miró e López-Vélez (2018), e neste sentido, a utilização das recomendações mundiais para casos dessa doença e seus vetores vêm diminuindo a incidência dessa zoonose pelo mundo. Faz-se necessária também a união política entre os países para incentivar a pesquisa e aplicação de novos medicamentos contra doenças tropicais como a leishmaniose (MITRA; MAWSON, 2017).

\section{RELATO DE CASO}

Foi atendido na Superintendência Hospitalar Universitária Veterinária da Universidade Federal Fronteira Sul, Campus Realeza, um cão de raça Chow-Chow, macho, sete anos, com queixa principal de sangramento nasal intermitente à 10 meses, com piora do quadro dias antes da consulta. $O$ paciente apresentou-se alerta, ofegante, andar cambaleante, com fraqueza muscular, claudicando e com ceratoconjuntivite. Durante a anamnese o tutor relatou que o cão viajou ao Tocantins por oito meses. Alimentado a base de ração e com as vacinas e vermífugação em dia. Ao exame físico apresentou mucosas hipocoradas, escore de condição corporal 4 (escala de 1-9), não visualizando nenhum ectoparasita. $\mathrm{Na}$ avaliação neurológica constatou-se ataxia e proprioceptivo leve em membro pélvico esquerdo. Foram coletadas amostras de sangue para realização de hemograma e avaliação bioquímica sérica.

O hemograma foi realizado em contador automático de células (Bio - 2900 Vet-Bioeasy Diagnostical- São Paulo, Brasil), registrando-se a contagem de leucócitos totais, número de hemácias, concentração de hemoglobina, volume corpuscular médio (VCM) e a concentração de hemoglobina corpuscular média (CHCM) e RDW (RedCell Distribuibution Width). O volume globular (VG) foi mensurado por método de microhematócrito. A avaliação morfológica foi realizada em esfregaço sanguíneo e capa leucocitária, corados com corante hematológico rápido tipo Romanowsky (Panótico Rápido LB- Laborclin Ltda, Pinhais, Brasil), e observados em microscópio óptico, com aumento de 1000x. A determinação da proteína plasmática total (PPT) foi realizada por refratometria, após a centrifugação do sangue em capilar de microhematócrito.

Todas as análises bioquímicas foram realizadas em analisador bioquímico semi-automático (BA 88A®- Mindray, Shenzhen Mindray Bio-Medical Eletronocs CO., LTD - Shenzhen, R.P. China), utilizando kits comerciais (Labtest $\AA^{\circledR}$ - Lagoa Santa/MG, Brasil) e soro controle universal (Qualitrol $1 \mathrm{H} \AA$, Labtest), conforme orientação do fabricante. Foram determinadas as concentrações séricas de uréia pelo método colorimétrico da Urease (Uréia $C E \circledast$ - Labtest, Lagoa Santa/MG, Brasil) e creatinina pelo método Picrato Alcalino (Jaffé) (Creatinina $\AA$, Labtest). A atividade enzimática da alanina aminotransferase (ALT) foi mensurada seguindo a metodologia Cinética UV - IFCC (ALT/GPT Liquiform Vet® - Labtest) e a fosfatase alcalina (FA) pelo método de Bowers e Mc Comb modificado (Fosfatase Alcalina Liquiform Vet $\AA$ - Labtest). 


\section{RESULTADO E DISCUSSÃO}

No hemograma observou-se redução de todas as séries celulares, caracterizando pancitopenia, em relação aos valores de referência para a espécie (RIZZI et al., 2010). Na avaliação da morfologia celular visualizaram-se hemácias em rouleaux, linfócitos reativos, raros rubrícitos policromáticos, hipocromia discreta, presença de bastonetes e estruturas compatíveis com Anaplasma sp em plaquetas. Em lâmina de capa leucocitária foram visualizadas formas amastigotas de Leishmania sp.em neutrófilos e monócitos (Figura 1).

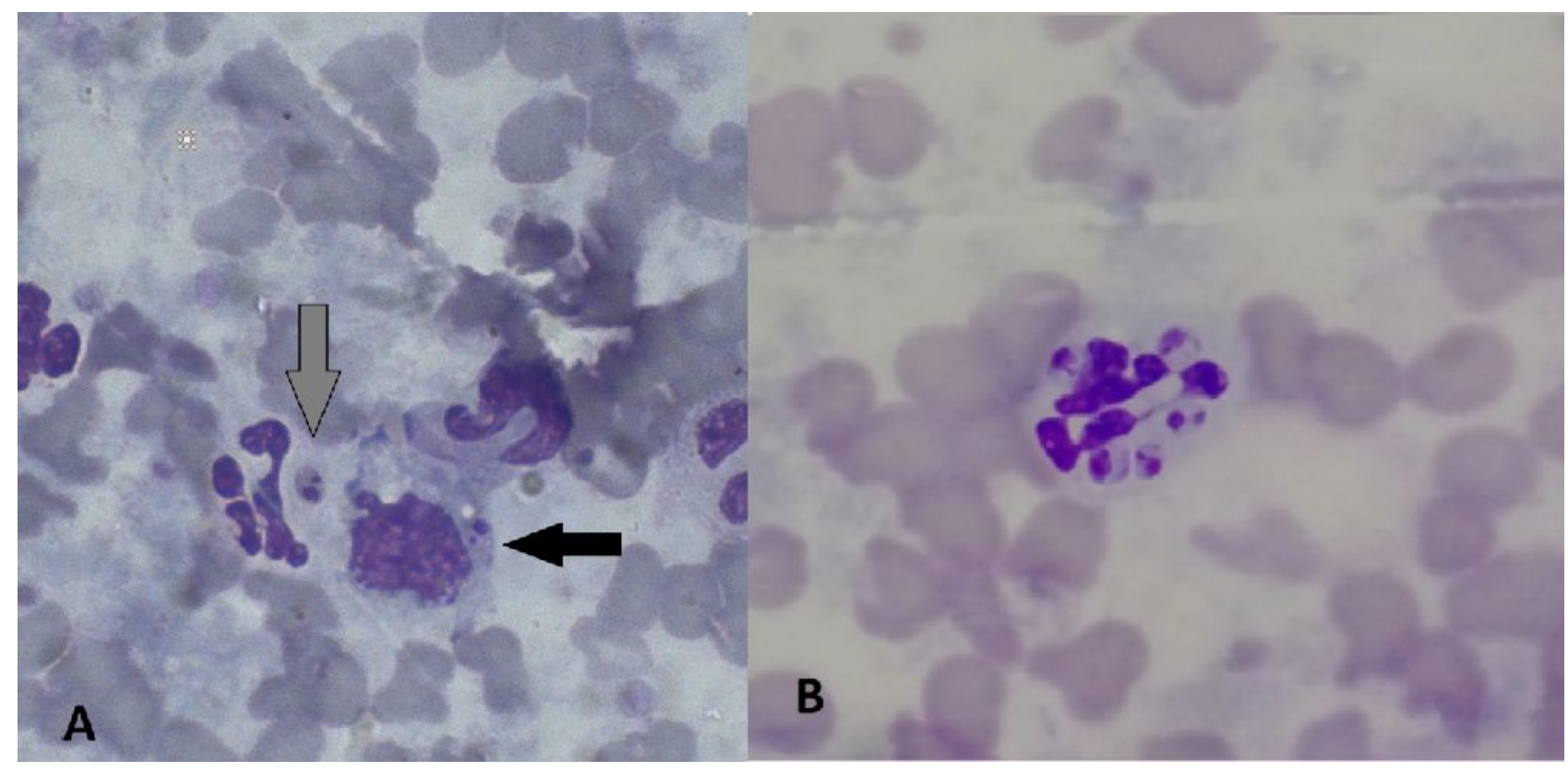

FIGURA 1: (A) Fotomicrografia de formas amastigotas de Leishmania sp. em neutrófilo (seta cinza) e monócito (seta preta) de sangue periférico de cão. (B) Fotomicrografia de neutrófilo com múltiplas formas amastigotas de Leishmania sp. no citoplasma. Aumento de 1000x, Realeza-PR. FONTE: Arquivo pessoal, 2019.

A detecção de amastigotas em sangue periférico é um achado incomum (FOGLIA MANZILLO et al., 2005). A defesa do sistema imune contra o parasita é conhecida pela atuação dos monócitos, porém os polimorfonucleares, em especial os neutrófilos mostram-se efetivos na eliminação do parasita durante a fase inicial da infecção e por isso têm papel importante nos estudos da LVC (FOGLIA MANZILLO et al., 2005). No eritrograma observou-se intensa anemia, com redução do número de hemácias $\left(1,68 \times 10^{6} / \mathrm{uL}\right)$, hemoglobina $(3,0 \mathrm{~g} / \mathrm{dL})$ e hematócrito $(11 \%)$. A anemia era do tipo normocítica (VCM=66fL) hipocrômica ( $\mathrm{CHCM}=27,2 \mathrm{~g} / \mathrm{dL})$, com RDW $(15,8 \%)$. A anemia é um achado comumente encontrado em animais sintomáticos para LVC e predomina o tipo normocitica/normocrômica (CORTESE et al., 2011; PALTRINIERI et al., 2016). A hipocromia observada justifica-se pela perda de sangue crônica que o animal apresentava, em decorrência da epistaxe, levando a espoliação dos estoques de ferro.

Além da perda sanguínea constante, anemia decorrente de processo inflamatório também é responsável pela diminuição da disponibilidade do ferro, devido ao sequestro desse mineral pelos macrófagos e redução da eritropoiese, pela 
menor síntese de hemoglobina, colaborando para o quadro de anemia (GANZ; NEMETH, 2009).

A perda de sangue intermitente pela epistaxe e ulcerações na pele são relatadas como causas de anemia em animais soropositivos para LVC, mas a infecção da medula óssea pelo parasita, hemólise, bem como aumento da uréia que interfere na função da eritropoietina e diminui a meia vida de eritrócitos também são possíveis causas do quadro anêmico desses pacientes (Da COSTA-VAL et al., 2007).

Além disso, Petanides et al. (2008) descrevem a epistaxe em animais positivos para leishmaniose como resultante da associação de mecanismos patogênicos relacionados às plaquetas, ulceração de mucosa nasal e hiperglobulinemia causando hiperviscosidade sérica.

A trombocitopenia é um achado frequente na LVC, acometendo de 40 a $50 \%$ dos animais positivos (BRAZ et al., 2015; FREITAS et al., 2017). Porém, não costuma ser tão intensa como no presente caso $(29.000 / \mathrm{uL})$, predominando reduções leves a moderadas (PALTRINIERI et al., 2016). A co-infecção por Anaplasma sp é a responsável pela intensidade das alterações hematológicas observadas, principalmente plaquetárias.

O leucograma indicou severa leucopenia (1.800 leucócitos/uL) o que impossibilitou a realização do exame diferencial dos leucócitos. Reis et al. (2006) também relatam que os animais apresentando sinais clínicos geralmente apresentam leucopenia, com diminuição de eosinófilos, monócitos e linfócitos, sendo esta última célula relacionada a presença de anticorpos circulantes.

De acordo com Silva e Winck (2018), a resposta leucocitária varia de acordo com a fase da doença. Braz et al. (2015), observaram que o valor médio dos leucócitos esteve dentro dos limites de normalidade, porém $27,3 \%$ dos casos em estudo apresentaram leucopenia.

A visualização de hemácias em rouleaux no esfregaço sanguíneo também foi observada por Rodrigues et al. (2018) e está relacionado com a elevação de globulinas. Freitas et al. (2017) encontraram hiperproteinemia em $82 \%$ dos casos, o que indica uma frequência alta desse achado laboratorial. Contudo, o caso em questão não apresentou aumento de proteínas plasmáticas $(5,8 \mathrm{~g} / \mathrm{dL})$. A LVC promove redução da relação albumina/globulina, com hipoalbuminemia e hiperglobulinemia (FREITAS et al., 2017), no presente caso não foi avaliada a concentração de albumina e globulinas, apenas a proteína plasmática total.

Cães positivos para LVC apresentando sinais clínicos demonstram aumento de IL-10, que é associado à maior suscetibilidade à doença, e aumento de INF-y no soro dos animais assintomáticos pode representar a cronicidade da doença (ROSÁRIO et al., 2018). Aumento de creatinina sérica (FREITAS et al., 2017), uréia e proteinúria caracterizam a lesão renal descrita na LVC (CÂMARA et al., 2017). No presente caso não evidenciou-se aumento de creatinina $(1,02 \mathrm{mg} / \mathrm{dL})$ ou uréia $(57,1 \mathrm{mg} / \mathrm{dL})$, o que indicou não ter havido comprometimento da função renal detectável até aquele momento, porém, não foi realizada avaliação da urinálise ou de imagem, que poderiam demonstrar presença de lesão renal de forma mais sensível.

Não foram observadas alterações nas enzimas hepáticas, a atividade enzimática da ALT (44U/L) e FA (110,2U/L) estavam dentro dos valores de referência para a espécie (RIZZI et al., 2010). Segundo Melendez-Lazo et al. (2017) observa-se com maior frequência em cães soropositivos para leishmaniose 
linfadenopatia, lesões de pele e perda de peso. Neste caso, o animal apresentava perda de peso e ceratoconjuntivite também descritos por Blavier et al. (2001).

Conforme citado por Reguera et al. (2016), a quimioterapia é considerada, apesar das variadas desvantagens que apresenta, o método de tratamento mais eficiente para a LVC. Não foram desenvolvidos ainda métodos que promovam a completa remoção do protozoário do organismo, sendo assim, a quimioterapia tem por objetivo enfraquecer o patógeno e diminuir a ocorrência da apresentação clínica da doença. Sendo necessária a realização de exames de carga parasitária, hematológicos e bioquímicos periódicos, que indiquem parâmetros como as funções hepática e renal do paciente. Até o momento apenas o Milteforan, é legalizado no Brasil para tratamento animal (BRASIL, 2016).

Para realizar o tratamento também é fundamental o uso de repelentes antiflebotomíneos para evitar contato entre vetor e fonte de infecção. No caso dos animais que não podem ser submetidos ao tratamento, é recomendada a eutanásia (BRASIL, 2016).

É dever do médico veterinário realizar a eutanásia nos casos devidamente justificados, observando os princípios básicos de saúde pública. Visto que, esta é uma doença de notificação compulsória, qualquer animal diagnosticado com a doença deve ser notificado aos órgãos públicos (CRMV/PR, 2015). No presente caso, assim que o responsável Médico Veterinário ficou ciente do diagnóstico de LVC no cão atendido, a vigilância sanitária da cidade foi informada, bem como o tutor do cão, que optou pela eutanásia do paciente em clínica particular.

Mesmo em regiões onde os casos de leishmaniose são esporádicos, a suspeita para a doença deve existir, já que a circulação de animais em viagens domésticas vem crescendo (BLAVIER et al., 2001). O uso de coleiras repelentes nos cães, vacinas e tratamento, são descritos e recomendados por alguns veterinários, porém a eutanásia ainda é uma das formas de controle mais utilizadas (ZUBEM; DONALÍSIO 2016). O estudo epidemiológico bem feito, associado a estratégias de controle da população de cães positivos para leishmaniose são fundamentais para o controle da doença tanto para humanos quanto animais, já que os cães são o principal reservatório urbano da doença (FARIA; ANDRADE, 2012; ROCHA et al., 2018; SILVA et al., 2017).

\section{CONCLUSÃO}

No paciente atendido, não predominaram as manifestações clínicas e laboratoriais clássicas da leishmaniose visceral, demonstrando a importância da pesquisa diagnóstica da doença em todos os animais oriundos de área endêmica que apresentem qualquer alteração clínica ou laboratorial sugestiva.

Apesar da visualização de amastigotas da Leishmania sp em sangue periférico ser incomum, o presente relato reforça a importância da avaliação morfológica em lâmina e da realização de capa leucocitária, principalmente nos animais leucopênicos.

\section{REFERÊNCIAS:}

ABRANTES, T.R.; WERNECK, G.L.; ALMEIDA, A.S.; FIGUEIREDO, F.B. Environmental factors associated with canine visceral leishmaniasis in an area with recent introduction of the disease in the State of Rio de Janeiro, Brazil. Cadernos de Saúde Pública, v. 34, n. 1, 2018. Disponível em: 
<http://www.scielo.br/pdf/csp/v34n1/1678-4464-csp-34-01-e00021117.pdf>. doi:10.1590/0102-311X00021117.

AKHOUNDI, M.; KUHLS, K.; CANNET, A.; VOTÝPKA, J.; MARTY, P.; et al. A historical overview of the classification, evolution, and dispersion of Leishmania parasites and sandflies. PLOS, Neglected Tropical Diseases, v. 10, n. 3, p. 1-40, 2016. Disponível em: <https://doi.org/10.1371/journal.pntd.0004349>. doi:10.1371/journal.pntd.0004349

BLAVIER, A.; KEROACK, S.; DENEROLLE, Ph.; GOY-THOLLOT, I.; CHABANNE, L.; et al. Atypical Forms of Canine Leishmaniosis. The Veterinary Journal, v. 162, p. 108-120, 2001. Disponível em: <https://doi.org/10.1053/tvjl.2000.0556>. doi:10.1053/tvjl.2000.0556

BRAZ, P. H.; SARTORETTO, M. C.; SOUZA, A. S.; MELO, F. M. G. Perfil hematológico de cães naturalmente infectados por Leishmania spp. Acta Veterinaria Brasilica, v. 9, n.1, p. 87-90, 2015. Disponível em: <https://doi.org/10.21708/avb.2015.9.1.5273>. doi: 10.21708/avb.2015.9.1.5273

BRASIL, ministério da agricultura, pecuária e abastecimento. Coordenação de Fiscalização de Produtos Veterinários- DFIP-SDA - CPV. Nota Técnica no 11/2016/CPV/DFIP/SDA/GM/MAPA. Dispõe sobre autorização do registro do produto Milteforan, indicado para o tratamento da leishmaniose visceral canina. 2016. Disponível em: <http://www.sbmt.org.br/portal/wpcontent/uploads/2016/09/nota-tecnica.pdf>. Acesso em 15 set 2018.

CÂMARA, C.S.; BALTAZAR, P.I.; GARCEZ, B.S. Alterações laboratoriais renais em cães com leishmaniose visceral naturalmente infectados. Pubvet Medicina Veterinária e Zootecnia, v. 11, n. 1, p. 35-39, 2017. Disponível em: $<$ http://dx.doi.org/10.22256/pubvet.v11n1.35-39>. doi: 10.22256/pubvet.v11n1.35-3

CRMV/PR- Conselho Regional de Medicina Veterinária do Estado do Paraná. Manual Técnico Leishmanioses Caninas. Curitiba - PR, v. 1, p. 46, 2015.

CORTESE, L.; TERRAZZANO, G.; PIANTEDOSI, D.; SICA, M.; PRISCO, M.; et al. Prevalence of anti-platelet antibodies in dogs naturally co-infected by Leishmania infantum and Ehrlichia canis. The Veterinary Journal, v. 188, p. 118-121, 2011. Disponível em:<https://www.ncbi.nlm.nih.gov/pubmed/20456989>. doi:10.1016 / j.tvjl.2010.03.015

Da COSTA-VAL, A.P.; CAVALCANTI, R.R.; GONTIJO, N.F.; MICHALICK, M.S.M.; ALEXANDER, B.; et al. Canine visceral leishmaniasis: Relation ships between clinical status, humoral immune response, haematology and Lutzomyia (Lutzomyia) longipalpis infectivity. The Veterinary Journal, v. 174, p. 636-643, 2007. Disponível em: <https://www.sciencedirect.com/science/article/pii/S1090023306002450>. doi:10.1016/j.tvjl.2006.11.006

DE TOMMASI, A.S.; OTRANTO, D.; DANTAS-TORRES, F.; CAPELLI, G.; BREITSCHWERDT, E.B.; et al. Are vector-borne pathogen co-infections complicating the clinical presentation in dogs?. Parasites \& Vectors, v. 6, n. 97, 2013. Disponível 
em:<http://www.parasitesandvectors.com/content/6/1/97>. doi:10.1186 / 1756-33056-97

FARIA, A.R.; ANDRADE, H.M. Diagnosis of canine visceral leishmaniasis: major technological advances and few practical applications. Revista Pan-Amazônica de Saúde, v. 3, n. 2, p. 45-57, 2012. Disponível em:<http://dx.doi.org/10.5123/S217662232012000200007>. doi:10.5123/S2176-62232012000200007

FOGLIA MANZILLO, V.; PIANTEDOSI, D.; CORTESE, L. Detection of Leishmania infantum in canine peripheral blood. Veterinary Record, v. 156, p. 151-152, 2005. Disponível em: <https://www.ncbi.nlm.nih.gov/pubmed/15715010>. doi:10.1136/vr.156.5.151

FREITAS, E.; MELO, M. N.; COSTA-VAL, A. P.; MICHALICK, M. S. M. Transmission of Leishmania infantum via blood transfusion in dogs: Potential for infection and importance of clinical factors. Veterinary Parasitology, v. 137, p. 159-167, 2006. Disponível em: <http://dx.doi.org/10.1590/0103-8478cr20141368>. doi:10.1590/01038478 cr20141368

FREITAS, M.V.M; BRUN, C.F.L.; RODRIGUES, M.C.; ALVES, G.B.B.; LEAL, A.F.; et al. Ocular diseases in dogs naturally affected by visceral leishmaniasis in Teresina, Piauí, Brazil. Ciência Rural, v. 47, n. 10, 2017. Disponível em $<$ http://www.scielo.br/scielo.php?script=sci_arttext\&pid=S0103-

$84782017001000504 \&$ lng=en\&tlng=en>. doi:10.1590/0103-8478cr20170029

GANZ, T.; NEMETH, E. Iron Sequestration and Anemia of Inflammation. Seminars in hematology, v. 46, n. 4, p. 387-393, 2009. Disponível em: <https://www.sciencedirect.com/science/article/pii/S0037196309000985>. doi:10.1053/j.seminhematol.2009.06.001

GIUDICE, E.; PASSANTINO, A. Detection of leishmania amastigotes in peripheral blood from four dogs - Short Communication. Acta Veterinaria Hungarica, v. 59, n. 2, p. 205-213, 2011. Disponível em:<https://www.ncbi.nIm.nih.gov/pubmed/21665574>. doi:10.1556/AVet.2011.003

GODOY, K.C.S.; ANTUNES, T.R.; BRAZ, P.H.; ASSIS, A.R.; OLIVEIRA, G.G.; et al. Comportamento dos marcadores bioquímicos de injúria hepática nos cães com leishmaniose visceral. PubVet Medicina Veterinária e Zootecnia, v. 11, n. 7, p. 670-675, $2017 . \quad$ Disponível em:< http://www.pubvet.com.br/artigo/3975/comportamento-dos-marcadores-

bioquiacutemicos-de-injuacuteria-hepaacutetica-nos-catildees-com-leishmaniosevisceral>. doi: 10.22256/PUBVET.V11N7.670 - 675

HANDMAN, E.; BULLEN, D. V. R. Interaction of Leishmania with the host macrophage. Trends in Parasitology, v. 18, n. 8, p. 332-334, 2002. Disponível em: <https://doi.org/10.1016/S1471-4922(02)02352-8>. doi:10.1016/S14714922(02)02352-8 
KAMHAWI, S. Phlebotomines and flies and Leishmania parasites: friends or foes? Trendes in Parasitology, v. 22, n. 9, p. 439-445, 2006. Disponível em: <https://www.ncbi.nlm.nih.gov/pubmed/16843727>. doi:10.1016/j.pt.2006.06.012

MAIA, C.; CAMPINO, L. Methods for diagnosis of canine leishmaniasis and immune response to infection. Veterinary Parasitology, v. 158, p. 274-287, 2008. Disponível em:<https://doi.org/10.1016/j.vetpar.2008.07.028>. doi:10.1016/j.vetpar.2008.07.028

MELÉNDEZ-LAZO, A.; ORDEIX, L.; PLANELLASA, M.; PASTORA, J.;SOLANOGALEGO, L. Clínicopathological findings in sick dogs naturally infected with Leishmania infantum: comparison of five different clinical classification systems. Research in Veterinary Science, v. 117, p. 18-27, 2017. Disponível em:<https://www.sciencedirect.com/science/article/abs/pii/S0034528816301850?via \%3Dihub> doi: 10.1016 / j.rvsc.2017.10.011

MENDONÇA, I. L.; BATISTA, J. F.; SCHALLIG, H.; CRUZ, M. S. P.; ALONSO, D. P.; et al. The performance of serological tests for Leishmania infantum infection screening in dogs depends on the prevalence of the disease. Journal of the São Paulo Institute of Tropical Medicine. v. 59, n. 39, p.1-10, 2017. Disponível em <http://dx.doi.org/10.1590/s1678-9946201759039>. 9946201759039

MIRÓ, G.; LÓPEZ-VÉLEZ, R. Clinical management of canine leishmaniosis versus human leishmaniasis due to Leishmania infantum: putting «One Health» principles into practice. Veterinary Parasitology, v. 254, p. 151-159, 2018. Disponível em:<https://www.sciencedirect.com/science/article/pii/S030440171830102X?via\%3Di hub>. doi:10.1016/j.vetpar.2018.03.002

MITRA; MAWSON. Neglected Tropical Diseases: Epidemiology and Global Burden. Tropical Medicine and Infection Diseases. v. 2, n. 36, 2017. Disponível em:<https://www.mdpi.com/2414-6366/2/3/36>. doi:10.3390/tropicalmed2030036

OLIVEIRA, V. V. G.; ALVES, L. A.; SILVA JUNIOR, V. A. Transmission routes of visceral leishmaniasis in mammals. Ciência Rural, v. 45, n. 9, p. 1-7, 2015. Disponível em: <http://dx.doi.org/10.1590/0103-8478cr20141368>. doi: 10.1590/0103-8478cr20141368

OLIVEIRA, V. V. G.; RAMOS, R. A. N.; RAMOS, C. A. N.; GUERRA, N. R.; MAIA, F. C. L.; et al. Molecular evidence of early vertical transmission of Leishmania (Leishmania) infantum in a dog. Ciência Rural, v. 47, n. 1. p. 1-4, 2017. Disponível em: <http://dx.doi.org/10.1590/0103-8478cr20160553>. doi:10.1590/0103$8478 \mathrm{cr} 20160553$

ORTIZ, R.C.; ANVERSA, L. Epidemiology of visceral leishmaniasis in Bauru, São Paulo, Brazil, 2004-2012: a descriptive study. Epidemiologia e Serviços de Saúde, v. 24, n. 1, p. 97-104, 2015. Disponível em: $<$ https://www.scielosp.org/scielo.php?script=sci_arttext\&pid=S223796222015000100097>. doi:10.5123/S1679-49742015000100011 
PALTRINIERI, S.; GRADONI, L.; ROURA, X.; ZATELLI, A.; ZINI, E. Laboratory tests for diagnosing and monitoring canine leishmaniasis. Veterinary Clinical Pathology, n. 1, v. 27, 2016. Disponível em: <https://www.ncbi.nlm.nih.gov/pubmed/27805725>. doi:10.1111/ vcp.12413

PETANIDES, T.A.; KOUTINAS, A.F.; MYLONAKIS, M.E.; DAY, M.J.; SARIDOMICHELAKIS, M.N.; et al. Factors Associated with the Occurrence of Epistaxis in Natural Canine Leishmaniasis (Leishmania infantum). Journal of Veterinary Internal Medicine, v. 22, p. 866-872, 2008. Disponível em <https://www.ncbi.nlm.nih.gov/pubmed/18564224> doi:1939-1676.2008.0129.x

REGUERA, R.; MORÁNA, M.; PÉREZ-PERTEJOA, Y.; GARCÍA-ESTRADA, C.; BALANA-FOUCE, R. Current status on prevention and treatment of canine leishmaniasis. Veterinary Parasitology, v. 227, p. 98-114, 2016. Disponível em <https://doi.org/10.1016/j.vetpar.2016.07.011> doi:10.1016/j.vetpar.2016.07.011

REIS, A. B.; MARTINS-FILHO, O. A.; TEIXEIRA-CARVALHO, A.; CARVALHO, M. G.; MAYRINK, W.; Parasite density and impaired biochemical/hematological status are associated with severe clinical aspects of canine visceral leishmaniasis. Research in Veterinary Science, v. 81, p. 68-75, 2006. Disponível em:<https://doi.org/10.1016/j.rvsc.2005.09.011>. doi:10.1016 / j.rvsc.2005.09.011

RIZZI, T.E.; MEINKOTH, J.H.; CLINKENBEARD, K.D.; Normal Hematology of the dog, In: Feldman B.F., ZINKE J.G.; JAIN N.C. (Ed.) Schalm's Veterinary Hematology. 5th ed.Baltimor, Estados Unidos: Lippicont Williams wikinscap 104, p.799-810, 2010.

ROCHA, M. A. N.; MATOS-ROCHA, T. J.; RIBEIRO, C. M. B.; ABREU, S. R. O. Epidemiological aspects of human and canine visceral leishmaniasis in State of Alagoas, Northeast, Brazil. Brazilian Journal of Biology, v. 78, n. 4, p. 609-614, 2018. Disponível em <http://dx.doi.org/10.1590/1519-6984.166622>. doi:10.1590/1519-6984.166622

RODRIGUES, K. S.; SILVA, A. M.; ALMEIDA, A. P.; SILVA, M. C.; VIANA, D. A.; et al. Leishmaniose canina na cidade de Caucaia, Ceará: Relato de Caso. Revista Pubvet v. 12, n. 8, p. 1-6, 2018. Disponível em: $<$ http://www.pubvet.com.br/artigo/4489/leishmaniose-canina-na-cidade-de-caucaiacearaacute-relato-de-caso>. doi: 10.31533/pubvet.v12n8a152.1-6

ROSÁRIO, C.J.R.M.; DOMINICI, M.F.; BRAGA, M.S.C.O.; LIMA, C.A.A.; PEREIRA, J.G.; et al. Quantificação da IL-10 e do INF-y em cães com ou sem sinais clínicos de infecção com Leishmania (Leishmania) chagasi. Pesquisa Veterinária Brasileira, v. 38, n. 1, p. 129-132, 2018. Disponível em:<http://www.scielo.br/pdf/pvb/v38n1/16785150-pvb-38-01-129.pdf>. doi:10.1590/S0100-736X2018000100020

SILVA, J.D.; MELO, D.H.M.; COSTA, J.A.G.; COSTA, D.F.; SILVA, R.B.S.; et al. Leishmaniose visceral em cães de assentamentos rurais. Pesquisa Veterinária Brasileira, v. 37, n. 11, p. 1292-1298, 2017. Disponível em: $<$ http://www.scielo.br/scielo.php?pid=S0100- 
736X2017001101292\&script=sci_abstract $>$.

doi:

$10.1590 / \mathrm{S} 0100$

736X2017001100016

SILVA C. M. H. S.; WINCK C. A. Leishmaniose visceral canina: revisão de literatura.

Revista da Universidade Vale do Rio Verde, v. 16, n. 1, p. 1-12, 2018. Disponível em <http://dx.doi.org/10.5892/ruvrd.v16i1.3383>. doi: 10.5892/ruvrd.v16i1.3383

ZUBEM, A.P.B.; DONALISIO, M.R. Difficulties in implementing the guidelines of the Brazilian Visceral Leishmaniasis Control Program in large cities. Cadernos de Saúde Pública, v. 32, n. 6, 2016. Disponível em: <http://dx.doi.org/10.1590/0102311X00087415>. doi:10.1590/0102-311X00087415 